This document is the Accepted Manuscript version of a Published Work that appeared in final form in Analytical Chemistry, copyright (C) American Chemical Society after peer review and technical editing by the publisher. To access the final edited and published work see http://pubs.ac$\underline{\text { s.org/doi/abs/10.1021/ac502627w }}$ 


\title{
Application of Solid Phase Microextraction for Quantitation of Polyunsaturated Fatty Acids in Bio- logical Fluids
}

\author{
Afsoon Pajand Birjandi ${ }^{\dagger}$, Fatemeh Sadat Mirnaghi1 ${ }^{\dagger \S}$, Barbara Bojko ${ }^{\dagger}$, Marcin Wąsowicz ${ }^{\ddagger}$ Janusz \\ Pawliszyn $^{\dagger *}$ \\ ${ }^{\dagger}$ Department of Chemistry, University of Waterloo, 200 University Avenue West, Waterloo, Ontario, N2L 3G1, Canada \\ ${ }^{\ddagger}$ Department of Anesthesia and Pain Management, Toronto General Hospital, Toronto, Ontario, M5G 2C4, Canada
}

\begin{abstract}
Development of a straightforward strategy for simultaneous quantitative analysis of nonesterified fatty acids (NEFA) species in biofluids is a challenging task because of the extreme complexity of fatty acid distri bution in biological matrices. In this study, we present a direct immersion solid phase microextraction method coupled to liquid chromatography-mass spectrometry platform (DI-SPME- HPLC-ESI -MS) for determination of unconjugated fatty acids (FA) in fish and human plasma. The proposed method was fully validated according to bioanalytical method validation guidelines. The LOD and LOQ were in the range of 0.5-2 and 5-12 ng/ml, respectively, with a linear dynamic range of 100 fold for each compound. Absolute and relative matrix effects were comprehensively evaluated and found to be in the acceptable range of $91-116 \%$. The affinity constant $\left(K_{a}\right)$ of individual FAs to protein albumin was determined to be $9.2 \times 10^{4}$ to $4.3 \times 10^{5} \mathrm{M}^{-1}$. The plasma protein binding (PPB \%) was calculated, and found to be in the range of $98.0-99.7 \%$ for different polyunsaturated fatty acids (PUFAs). The PUFAs under study were found at a high concentration range in fish plasma, whereas only a few were within quantification range in control human plasma. The method was successfully applied for monitoring PUFA changes during the operation in plasma samples obtained from patients undergoing cardiac surgery with the use of cardiopulmonary bypass (CPB). The most significant contribution induced by surgery was noticed in the concentration level of a-linolenic acid (18:3, ALA), arachidonic acid (20:4, AA), and docosahexanoic acid (22:6, DHA) soon after administration of CPB in all cases.
\end{abstract}


Fatty acids (FA) are essential components of living cells and important substrates that play a critical role in mammalian energy metabolism. Fatty acids are combined as the building blocks of more complex lipids through ester or amide bonds. These lipids will form the lipid bilayer membrane compositions, lipoporoteins and liposomes. Esterified fatty acids act as suppliers of chemically stored energy, building blocks of cellular membranes by esterification into phospholipids and involving in signal transducers pathways. ${ }^{1}$ They are continuously produced, integrated into lipids, and degraded in the $\beta$-oxidation pathway and citric acid cycle. ${ }^{2}$ On the other hand, fatty acids also exist in free form, (nonesterified fatty acids; NEFA) in the biological media. Alterations in the metabolism of this portion is of specific interest since it is established to be associated with pathological conditions and observed with numerous disorders, such as obesity, ${ }^{3}$ insulin resistance, ${ }^{4}$ diabetes mellitus, ${ }^{5}$ or metabolic syndrome. ${ }^{6}$

Accurate determination of the composition of free fatty acids (FFA) in different biological matrices is a predominant problem in total fat extraction. The most common procedures used to measure FFA concentration consist of multiplestep methods, including (a) an extraction procedure to isolate lipids from the sample bulk, (b) separation of FFA from the rest of the lipids using conventional methods such as thin-layer chromatography (TLC) and/or solid phase extraction (SPE), ${ }^{7,8}$ (c) derivatization of FFA to fatty acid methyl esters (FAMEs), ${ }^{9,10}$ and (d) a final chromatographic method for differentiation of individual fatty acid species. ${ }^{11}$ The chromatographic determination of FAMEs is by far mostly done using capillary gas chromatography (GC, $)^{12-14}$ and less frequently by high performance liquid chromatography (HPLC). ${ }^{9,15,16}$
Lipid analysis deals with enormous sample complexity. In order to obtain satisfactory results, the extraction of lipids from complex biological matrices, which aims at removal and isolation from interfering agents such as proteins, saccharides, or other small molecules, is usually indispensable before the analysis. Therefore, a broad range of extraction techniques are currently used for this purpose. ${ }^{17}$ However, the most common extraction approaches have been mainly based on solvent extraction so far. The most popular extraction methods for lipids include the traditional Folch method, ${ }^{18}$ or a modified Folch method ${ }^{13}$ that employs a solution of chloroform/methanol $(2: 1, v / v)$, or the commonly called Bligh and Dyer method, in which a chloroform/ methanol/water mixture is used to extract the lipids. ${ }^{19}$ Moreover, exhaustive Soxhlet extraction is probably the most commonly used technique for the extraction of fats and oils from food matrices. ${ }^{20}$ More modern methodologies take advantage of solid-phase extraction (SPE) for fatty acid extraction, ${ }^{21}$ typically using aminobonded phase and C18 bonded-phase columns. ${ }^{22}$ In comparison to liquid-liquid extraction (LLE) methods, these procedures are quite fast, minimizing volumes of organic solvents and leading to good recovery and higher reproducibility. However, most of the proposed SPE protocols offer the application of derivatization or liquid extraction followed by solvent evaporation and reconstitution procedure. ${ }^{23}$ In addition, the limitations may include clogging of cartridges when handling complex matrixes such as plasma or tissue. Considering the exhaustive nature of SPE, when dealing with a large volume of samples the recovery will be significantly reduced due to limitations with breakthrough volume and the low peak capacity of SPE cartridges. ${ }^{24-26}$

In contrast, among all extraction techniques, solid phase microextraction (SPME) is sampling and sample preparation technique characterized 
by simplicity, reproducibility and non-exhaustive nature of the extraction process when very small sorbent volume is used. The benefits of SPME for highly complex matrices such as biological samples have been already discussed elswhere. ${ }^{27-32}$ Headspace SPME (HS-SPME) has been previously reported for determination of short chain volatile fatty acids, including the $\mathrm{C}_{2}-\mathrm{C}_{7}$ carbon chain or their ethyl esters from waste water. ${ }^{33,34}$ However, extraction of long chain fatty acids in biological and nutrition analysis is a very challenging goal due to their hydrophobicity, perceived abundance as plasticizers, ubiquity in the environment, great tendency to bioconcentrate, vast distribution in conjugated forms in cellular structure, very high affinity to biological proteins such as albumin, and a high risk of matrix effect encounters. The main goal of this study is to address the above mentioned challenges for unbiased high throughput quantification of "total" and "free" concentration of nonesterified fatty acids in the complex biological media via optimization and validation of a SPME assay followed by HPLC-ESI-MS. The method involves a simple SPME protocol with no necessity of using halogenated solvents or chemical derivatization approaches that use highly reactive reagents. In the proposed method, entire procedure is simplified to immersion of SPME fiber into the biological fluids which allows for extraction of the NEFA without interrupting the lipoproteins or cell membrane lipids. This is in contrast to the commonly used liquid based methods which only provide estimation of total concentration of fatty acids due to the disruption of protein-bound fractions and complex lipid structures such as lipoproteins by organic solvents. Therefore, as the SPME extraction is non-exhaustive, the natural balance in the investigated system is not disturbed and the obtained results provide information about the actual equilibrium in the sample.
The possibility of encountering matrix effect in HPLC-ESI-MS analysis of human and fish plasma was evaluated using different experimental approaches. The proposed method was fully validated according to the bioanalytical method validation guidelines. The final protocol was applied to monitor PUFA changes in plasma obtained from a group of patients during cardiac surgery with the use of cardiopulmonary bypass (CPB) and to quantify level of the PUFAs in fish plasma.

\section{EXPERIMENTAL SECTION}

Plasma samples Plasma samples were obtained from seven patients during cardiac surgery involving the use of cardiopulmonary bypass (CPB). Blood samples were taken on a sampling schedule order at 5, 10 and 15 minutes before initiation of the infusion; 5 minutes after chest opening and 5 minutes before commencing CPB, following by frequent sampling every 30 minutes during CPB; and 5, 60 and 120 minutes after chest closure. ${ }^{35-38}$ Perioperative care has been provided to all the patients as described previously. ${ }^{39}$ The study approval was obtained from Toronto General Hospital/University Health Network and University of Waterloo Research Ethics Boards. All the patients signed the consent to participate in the study.

Fish plasma samples were also collected from White Sucker (Catostomus commersoni) at Lake Superior (Provincial Park, ON) due to their widespread availability in the watershed. Blood was collected by caudal puncture with a heparincoated needle and syringe $(5 \mathrm{cc})$ and centrifuged at $10000 \mathrm{rpm}$ for $4 \mathrm{~min}$ to separate plasma. All plasma samples was transferred to a cryovial, snap-frozen in liquid nitrogen and stored at -80C until analysis. Animal care and all investigative procedures adhered to the guidelines of the Office of Research Ethics, University of Waterloo (AUPP:10-17) and the Canadian Council of Animal Care. 
Optimization of SPME Procedure C18 and mixmode fibers (Supelco, Bellefonte PA) were compared during the preliminary stage of extraction phase selection. In order to determine the extraction efficiency and reproducibility of the SPME coatings, a phosphate buffered saline (PBS) at ph 7.4 was spiked with authentic standards of fatty acids for a concentration of 0.1 $\mu \mathrm{g} / \mathrm{ml}$. Prior to use, all fibers were preconditioned by 30 minutes agitation in a methanol: water solution $(1: 1, v / v)$ in order to activate the silanol groups of the stationary phase. The analytes spiked in plasma were pre-incubated in room temperature for 60 min prior extraction to allow establishment ofprotein binding, knowing that the binding rate of fatty acid to albumin is rapid. ${ }^{40}$ The SPME experiment was performed by immersing the fibers into $1 \mathrm{~mL}$ of sample aliquots for 60 min extraction time with $800 \mathrm{rpm}$ orbital shaking (model DVX-2500, VWR International, Mississauga, ON). Immediately after extraction, fibers were rinsed in purified water for $10 \mathrm{~s}$ to remove any remains of biological material from the coating surface, followed by 60 minutes desorption in $1 \mathrm{ml}$ acetonitrile with agitation (1000 rpm) . Extracts were further injected to HPLC-ESI-MS system for analysis. Percentage of extraction efficiency (or percent absolute recovery) was calculated as the ratio of the amount extracted versus total amount of fatty acid spiked $\times 100 \%$. Development of more detailed experimental protocol can be found in the Supporting Information.

LC-ESI-MS Operating Conditions All samples were analyzed using an HPLC-ESI-MS system consisting of two Varian 212-LC pumps (Walnut Creek, CA), a Prostar 430 autosampler, and a 500-MS ion trap mass spectrometer (Varian, Palo Alto, CA). Data acquisition and processing were performed using Varian MS Workstation software (Version 6.6). Chromatographic separation was performed on an Ascentis ${ }^{\circledR}$ Express C18 RP-
LC column $(2.1 \times 150 \mathrm{~mm}, 2.7 \mu \mathrm{m})$. The binary gradient run consisted of eluent A $(90 \%$ water, $10 \%$ Methanol) and B (80\% methanol, 20\% acetonitrile) at room temperature with a flow rate of $0.3 \mathrm{~mL} / \mathrm{min}$ and $10 \mu \mathrm{l}$ injection volume. Optimal separation was achieved using the following solvent gradient elution: Mobile B starts with $60 \%$ holding for $1 \mathrm{~min}$ ( $\min 0-1$ ), increasing to $95 \%$ (min 1-2), increasing to $100 \%$ (min 2-5), held for one minute ( $\min 5-6$ ), then ramped back to $60 \%$ over thirty seconds, followed by two more minutes of re-equilibration resulting in a total run time of eight minutes. All fatty acids were analyzed at negative ionization mode (forming [M$\left.\mathrm{H}^{-}\right)$and were monitored in full scan mode. The optimum MS parameters were as follow: capillary voltage: $90-110 \mathrm{~V}$, RF loading of $80-90$, ion spray voltage $-4500 \mathrm{~V}$ and drying gas temperature $400^{\circ} \mathrm{C}$. The Figure S-1 in Supplementary Information shows the examples of XIC chromatograms of fatty acids from human plasma extract.

\section{Determination of Protein Affinity Constant} $\left(K_{a}\right)$ The extraction recovery of NEFAs was measured at different human serum albumin (HSA) concentrations to determine if variations in HSA level affect the free concentration and consequently the extraction recovery of studied fatty acids. Human serum albumin (essentially fatty acid and globulin free) was dissolved in PBS buffer solution $(\mathrm{pH} 7.4)$ to reach protein concentrations of $0,5,10,20,30,40,50,60,70$ and 100 $\mathrm{g} / \mathrm{L}$, These solutions were then spiked with fatty acids standards to reach the concentration of 3 $\mu \mathrm{g} / \mathrm{mL}$ in all solutions. The study was conducted in duplicate.

Determination of plasma protein binding: investigation of free and total concentrations The determination of plasma protein binding by SPME is based on the quantification of the free concentration of ligand in the presence of 
plasma proteins. ${ }^{41-47}$ In order to have a better understanding of plasma protein binding, the calibration curves were not only constructed in PBS and plasma, they were also constructed in standard human serum albumin solution (essentially fatty acid free) to mimic the plasma with only albumin as binding agent. Linearity was verified by analyzing spiked plasma samples at the concentrations of $0,1,2,3,4,5$, and $7 \mu \mathrm{g} / \mathrm{mL}$, with three replicates in each point. After incubation allowing for protein binding equilibrium (60 min), extractions from PBS, serum albumin and plasma samples were performed under the same conditions using SPME fibers. Batch-to-batch precision was determined according to Matuszewski et al. ${ }^{48}$ Five different sources of human plasma at all concentrations ( $\mathrm{n}=3$ for each point) were utilized for the construction of standard addition calibration curves.

\section{RESULTS AND DISCUSSIONS}

HPLC-ESI-MS Electrospray ionization in negative mode was chosen because fatty acids form $[\mathrm{M}-\mathrm{H}]^{-}$quasimolecular ions due to their carboxylic acid moiety. Disregard of the applied collision energy level, the product ion spectrum of $[\mathrm{M}-\mathrm{H}]^{-}$in MRM mode was also dominated by the unfragmented deprotonated molecular ions of a low sensitivity. The lack of production of detectable fragments in ESI-MS/MS has been already described for underivatized FAs. ${ }^{49}$ Peak widths of 4-6 $s$ were found and chromatographic resolution could be achieved in a total run time of $8 \mathrm{~min}$. Based on the chromatographic method, a two-carbon increase in fatty acid chain length increases the retention time by $\sim 1.8$ min, whereas introduction of a double bond, decreases retention time by $\sim 0.7 \mathrm{~min}$.

SPME method development The experimental standard procedure was followed based on previously published SPME protocol for method vali- dation. ${ }^{50}$ The properties of the $\mathrm{C} 18$ biocompatible fibers was described in details elsewhere. ${ }^{51,52}$

Extraction: The extraction time profile was obtained in both PBS and plasma. The results regarding to extraction efficiency obtained in PBS solution as a matrix free media are described in Supporting Information (section 2.2). The initial stages of extraction time profile for all fatty acids under study were similar in both PBS and plasma meaning that the equilibrium was achieved in both media within first two hours. However, after four hours of extraction in plasma, the extracted amount started to increase reaching second plateau after 10 hours and remaining constant for up to more than 18 hours extraction (Figure S-2, Supporting Information). Checking the physical appearance of the fiber, a jelly-like attachment around the fiber was observed after 4 hours extraction with vortex agitation. The initiation of protein attachment was observed around the metal part of the rod fibre which is in contact with plasma during long time aggressive agitation. This effect could be explained as a result of protein attachment to the surface of metal. The coating biocompatibility was tested several times and has been reported previously; ${ }^{51,52}$ hence it is anticipated that this effect is only visible for longer contact of fibre with aggressively agitated sample. Therefore, extraction time must be long enough to meet sensitivity requirement, and short and gentle to avoid the initiation of protein aggregation around the fiber. Therefore, the use of $800 \mathrm{rpm}$ orbital shaking, which is a less aggressive agitation approach compared to vortex agitation, prevent the protein aggregation occurrence. The required time to reach equilibrium extraction in plasma for all nine compounds was less than 120 minutes; therefore, 2 hours was chosen as the optimum extraction time for the entire study. The evaluation of washing, desorption and carry over are 
discussed in Supporting Information (section 2.1).

\section{Evaluation of matrix effect and ionization sup-} pression Matrix effect can be considered as the Achilles heel of quantitative mass spectrometric analysis. Matrix effect occurs when matrix molecules coelute with the analyte of interest thus altering the ionization efficiency of the electrospray interface. ${ }^{48}$ Therefore, it is essential to employ appropriate strategies to minimize the ionization suppression or enhancement phenomenon associated with matrix effect. SPME, however, is a clean extraction method, where the biocompatibility of the polymer coating prevents extraction of macromolecules and other matrix components. Moreover, non-exhaustive extraction by SPME fiber applies not only to the analyte of interest, but also possible interfering compounds, thus eliminating or significantly minimizing competition in ionization process. In this study, the matrix effect was assessed using two different approaches including i) absolute matrix effect evaluation using the post-extraction spiked method described by Matuszewski et al. ${ }^{53}$ and ii) the sample extract dilution method. ${ }^{54}$ Calculation of absolute matrix effect involved relating the peak area obtained from a neat solvent spike with a known concentration (S2) to the peak area of the blank extract, which is spiked with the same concentration of analyte standard after extraction (S1)

$M E \omega=\left(\frac{\operatorname{arnsi2}}{\operatorname{arns} 1}\right) \times 10 \mathrm{~d}$

(Equation 1)

ME values larger than $120 \%$ and smaller than $80 \%$ represent significant ionization enhancement or suppression for a given analyte. For this study, matrix effect (ME) values are reported in Table 1. Utilizing the proposed SPME method, no absolute matrix effect was observed. Additional

proof is provided by evaluation of matrix effect using the Sample Extract Dilution Method (Supporting Information, Section 2.3).

\section{Determination of albumin affinity constant by} SPME In plasma, fatty acids are soluble in concentrations up to about $1 \mu \mathrm{M}$. Owing their low solubility in plasma, FAs require a transporter to increase their concentration in vascular and interstitial compartments. Human serum albumin (HSA) is the transport vehicle for free fatty acids and the main FA-binding protein in extracellular fluids ${ }^{55}$ which binds with approximately 0.12 mol per mole protein, under normal physiological condition. ${ }^{56}$ Further increase of NEFA concentration increases the bound NEFA proportion, accordingly. ${ }^{40}$ Because SPME extraction occurs via free concentration, the study of plasma protein binding (PPB\%) and the effect of albumin concentration on fatty acid recovery by SPME seemed crucial for this study. The recovery profile of NEFAs was measured at different HSA concentrations. Amount of FAs extracted from spiked standard human albumin solutions were plotted against the protein concentrations $\left(C_{p}\right)$, and the $K_{a}$ was determined by fitting equation 2 through the data points. ${ }^{57}$

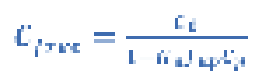

(Equation 2) 
Where, $\mathrm{C}_{0}$ is the amount extracted at a protein concentration of 0 , and $f_{\text {up }}$ is the unoccupied fraction of protein. In this experiment, $f_{u p}$ is approximately equal to 1 because the total protein concentration (5-100 g/L) was much higher than the spiked fatty acid concentration ( $3 \mu \mathrm{g} / \mathrm{L}$ ). Figure 1 shows the absolute recovery calculated for each individual protein concentration. The reference range for albumin concentrations in human blood plasma is 34 to $54 \mathrm{~g} / \mathrm{L}$. In the exaggerated range of $\mathrm{HSA}(<20$ and $>70 \mathrm{~g} / \mathrm{L}$ ), protein concentration affects the recovery of fatty acids by changing its free concentration equilibrating with the SPME coating. However, these extreme values are not expected in plasma samples except of hyper- or hypoalbuminemia and in this study they are only considered in order to evaluate fiber performance. As the results show in Figure 1 , absolute recovery is constant within the physiological range of HSA concentration (30-60 g/L). Accordingly, the mean calculated $K_{a}$ values from this experiment were in the range $9.2 \times 10^{4}$ to $4.3 \times 10^{5} \mathrm{M}^{-1}$ (Table 2 ). Affinity constant $\left(K_{a}\right)$ was reported to be dependent on the fatty acids carbon chain length; it increases with an increase in length and decrease of the number of double bounds in studied fatty acids. ${ }^{40}$ The obtained results for the affinity constant using the SPME method follow the same pattern and correspond very well with literature values, ${ }^{55,58}$ which proves the validity of the proposed SPME method for the study of free concentration and protein binding of nonesterified fatty acids in biological systems.
Plasma protein binding (PPB \%) Binding equilibrium studies for long-chain fatty acids to serum albumin are complicated because of the low ligand solubility. The aim of this part of the study was to investigate binding equilibria of long-chain fatty acids in human blood plasma under varying conditions and to compare the results with observations on the affinity constant to serum albumin, which were discussed in the previous section. Plasma protein binding determination by SPME method has been reported based on the measurement of bioactive unbound concentrations of the ligand, which is often referred as the free concentration in presence of plasma protein. ${ }^{59}$ 


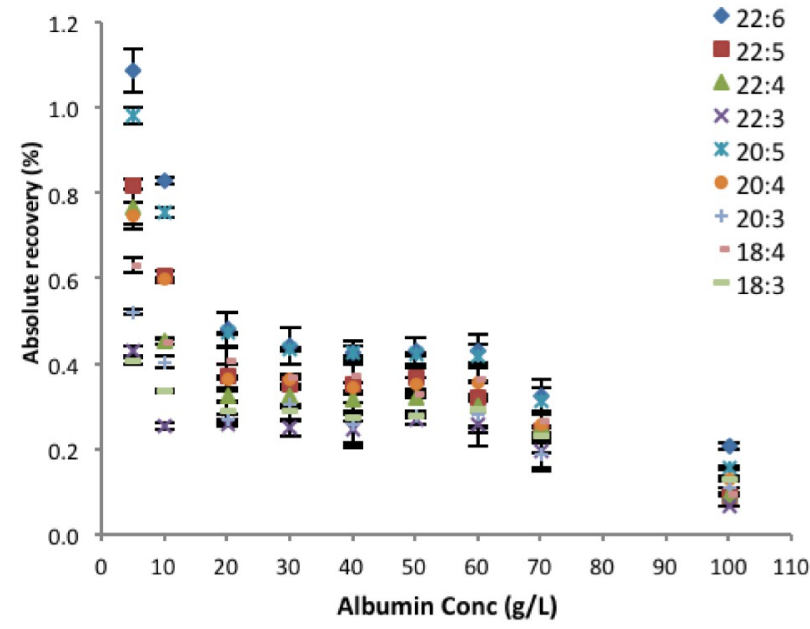

Figure 1 Albumin concentration-dependent NEFA recovery; yaxis represents the extraction recovery percentage of fatty acids from solutions with different albumin concentrations after $2 \mathrm{hr}$ of extraction.

Briefly, the percentage of binding to plasma proteins (PPB) is calculated from the total and free concentrations of analyte:

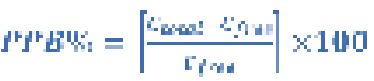

Equation 3

Where $C_{\text {total }}$ is the total concentration of ligand and $\mathrm{C}_{\text {free }}$ is the free concentration of ligand in plasma. Considering that the total ligand concentration is directly proportional to the slope of the matrix-free calibration curve in PBS, and the free concentration is directly proportional to the slope of matrix match calibration; ${ }^{59}$ Equation 3 becomes:

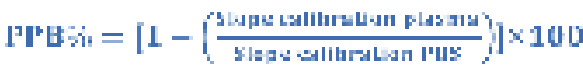

Equation

4

In order to better understand the FA binding mechanism, the matrix match calibration curve was not only constructed in plasma, it was also constructed in HSA standard buffered solutions (35 g/L). Using the standard slopes obtained from these experiments, Equation 4 was applied for determination of PPB values of nine PUFAs under study in both media; results are presented in Table 1. The present observations demon- strate that there are no significant variations in PPB values determined by SPME method using different approaches, and it correlates well with average literature values. ${ }^{40,58,60}$

\section{Fatty acids quantification in plasma samples} One of the most important elements of assay validation is the evaluation of the effect of matrix on the results of quantitative determination of metabolites in biological fluids. ${ }^{61}$ Therefore, due to the limited availability of plasma samples it was essential to compare the reproducibility of standard addition calibration in different plasma lots under the same extraction and chromatographic conditions. The precision and accuracy values obtained in a single plasma lot using the proposed SPME method ranged from 5 to $10 \%$, and 95 to $110 \%$, respectively. Moreover, when the same validation was attempted in five different plasma lots, the precision values were persuasive (7-14\%) under identical conditions. The inter-lot and intra-lot reproducibility is reported in Table 1 . The very small variability of slopes of calibration curves obtained from five different plasma sources is a direct indicator of assay reproducibility and serves as a good quantitative indicator of the absence of a relative matrix effect in the proposed SPME method. For quantification of the fatty acids under study, the mean calibration curve, obtained from five different plasma lots, was employed to extrapolate and quantify the unknown amount of PUFAs in human plasma. Due to the limited availability of fish plasma, the standard addition method was performed in an individual lot of pooled fish plasma (three replicates for each concentration point). Data are summarized in Table 2.

Comparison of NEFA composition in fish and human plasma In general, the mean of standard calibration line slopes determined in human control plasma and fish plasma are similar for all fatty acids, which serves as an excellent measure 
of absence of a relative matrix effect not only within the same individual, but also between individuals. The observed differences of the $\mathrm{Y}$-axis intercept of the calibration equations clearly indicate that the initial level of fatty acids is considerably higher in fish. Total plasma NEFA in the study of White Sucker species ranged from 0.76 to $11.13 \mu \mathrm{g} / \mathrm{mL}$, whereas for control human plasma only arachidonic acid was detectable in the quantification range. The amounts of DHA and EPA were significantly higher in fish plasma with a $p$ value of 0.002 and 0.007 , respectively, though the concentration level of arachidonic acid was not statistically different between these two subjects with a nearly non-significant $p$ value (0.78). These results are consistent with the erstwhile work done on the White Sucker fish, which indicated higher DHA and EPA, and lower AA. ${ }^{62-64}$ 
Table 1 Evaluation of albumin affinity constant and \% plasma protein binding

\begin{tabular}{lccccccccc}
\hline & DHA & EPA & ADR & DTA & EPA & ARA & ETA & SDA & ALA \\
\hline Matrix Effect (\%) & $103 \%$ & $96 \%$ & $88 \%$ & $91 \%$ & $116 \%$ & $110 \%$ & $93 \%$ & $112 \%$ & $116 \%$ \\
$\begin{array}{l}\text { Albumin affinity con- } \\
\text { stant (M }\end{array}$ & $9.2 \times 10^{4}$ ) & $2.1 \times 10^{5}$ & $2.2 \times 10^{5}$ & $4.3 \times 10^{5}$ & $1.2 \times 10^{5}$ & $1.5 \times 10^{5}$ & $2.9 \times 10^{5}$ & $2.0 \times 10^{5}$ & $2.2 \times 10^{5}$ \\
$\begin{array}{l}\text { Human serum albu- } \\
\text { min PPB (\%) }\end{array}$ & 97.9 & 98.6 & 99.1 & 99.4 & 98.0 & 98.6 & 99.2 & 99.5 & 99.6 \\
$\begin{array}{l}\text { Plasma PPB (\%) } \\
\begin{array}{l}\text { Intra-batch repro- } \\
\text { ducibility (\%) }\end{array}\end{array}$ & 98.3 & 99.3 & 99.5 & 99.7 & 98.7 & 98.8 & 99.6 & 99.5 & 99.6 \\
$\begin{array}{l}\text { Inter-batch repro- } \\
\text { ducibility (\%) }\end{array}$ & 9 & 10 & 6 & 9 & 5 & 5 & 6 & 6 & 10 \\
\hline
\end{tabular}

PPB values are found with ( $\pm 0-0.2)$ standard deviation and the correlation coefficient (CV) lower than $15 \%$.

Table 2 Linear dynamic range of fatty acid concentration in human and fish plasma samples

\begin{tabular}{|c|c|c|c|c|c|}
\hline & $\begin{array}{l}\text { Human plasma cali- } \\
\text { bration }(\mathrm{n}=5)^{1}\end{array}$ & $\begin{array}{l}\text { Lot-to- } \\
\text { lot CV } \\
(\%)^{2}\end{array}$ & $\begin{array}{c}\text { Fish plasma calibra- } \\
\text { tion }^{4}\end{array}$ & $\begin{array}{c}\text { Human } \\
\text { plasma } \\
\text { conc. }(\mu \mathrm{g})^{3}\end{array}$ & $\begin{array}{l}\text { Fish plasma } \\
\text { conc. }(\mu \mathrm{g})^{5}\end{array}$ \\
\hline DHA & $y=0.0041 x+0.67$ & $5 \%$ & $y=0.0044 x+26.9$ & LOD & $11.1 \pm 0.2$ \\
\hline DPA & $y=0.0037 x+10.5$ & $4 \%$ & $y=0.0039 x+16.6$ & ND & $6.6 \pm 0.1$ \\
\hline ADR & $y=0.0019 x+1.6$ & $5 \%$ & $y=0.0019 x-0.5$ & ND & LOD \\
\hline DTA & $y=0.0018 x+3.3$ & $3 \%$ & $y=0.0022 x+4.3$ & ND & $3.0 \pm 0.1$ \\
\hline EPA & $y=0.0041 x+4.7$ & $3 \%$ & $y=0.0041 x+13.1$ & LOD & $9.0 \pm 0.1$ \\
\hline ARA & $y=0.0036 x+1.7$ & $3 \%$ & $y=0.0027 x+1.04$ & $2.9 \pm 0.1$ & $2.7 \pm 0.1$ \\
\hline ETA & $y=0.0026 x+0.8$ & $4 \%$ & $y=0.0025 x+0.4$ & $1.2 \pm 0.1$ & $1.1 \pm 0.01$ \\
\hline STD & $y=0.0034 x+1.26$ & $4 \%$ & $y=0.0044 x+1.1$ & ND & $0.76 \pm 0.01$ \\
\hline ALA & $y=0.0012 x+1.5$ & $5 \%$ & $y=0.0011 x+5.2$ & $2.8 \pm 0.2$ & $1.6 \pm 0.1$ \\
\hline
\end{tabular}


Our results confirm the well known fact that fish is an omega-3 rich food choice because of its primary source of essential fatty acids and demonstrate that presented analytical protocol can be used for assessing the nutritional value of fish products in simple and relatively fast manner.

Clinical data analysis Using the validated SPME method, fatty acid changes were monitored in seven patients. As the results indicate in Figure 3, the major changes in concentration of unsaturated fatty acids during the surgery refer to the period when the patient is placed on CPB (Table 2). Pre-operative or intra-operative metabolic changes in cardiac surgical patients are widely discussed in the literature. ${ }^{65-67}$ From a metabolic point of view, the cardiovascular response to increased metabolic demands after cardiac surgery may lead to enhanced glucose and down regulated free fatty acid (FFA) metabolism; yet the heart can use several substrates, among which free fatty acids (FFAs) and glucose are the major sources. ${ }^{65}$ Having known this fact, the reason behind fatty acid elevation right after commencing bypass could be clearly explained. The alteration in metabolic profile of ALA and its metabolites was already reported and discussed in the studied group of patients and current targeted analysis confirms previous findings. ${ }^{38}$ 
Patient \# 1

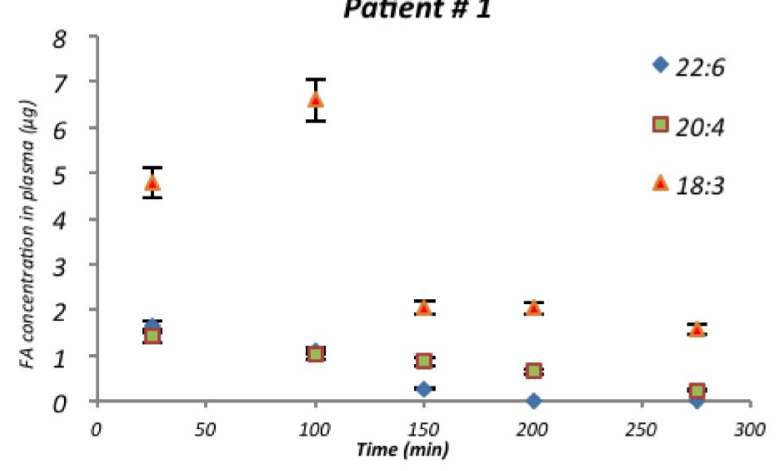

Patient \#3
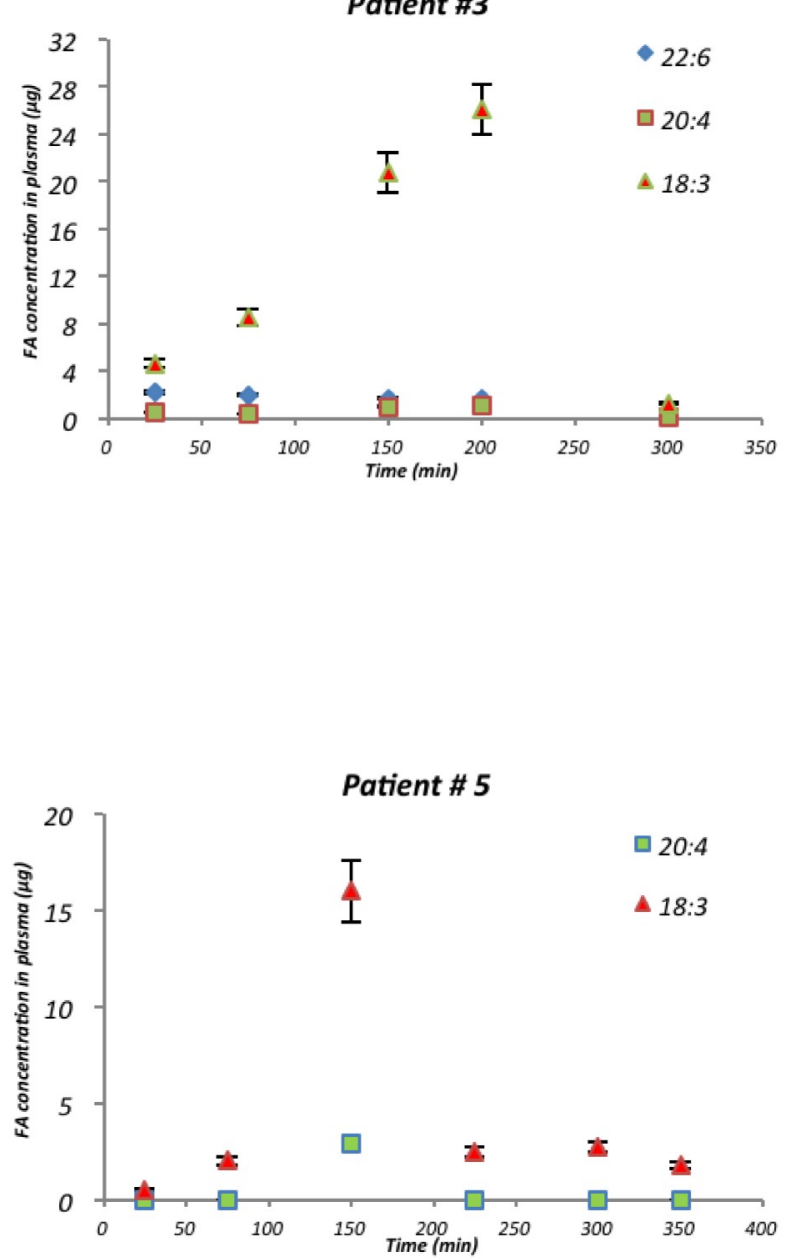
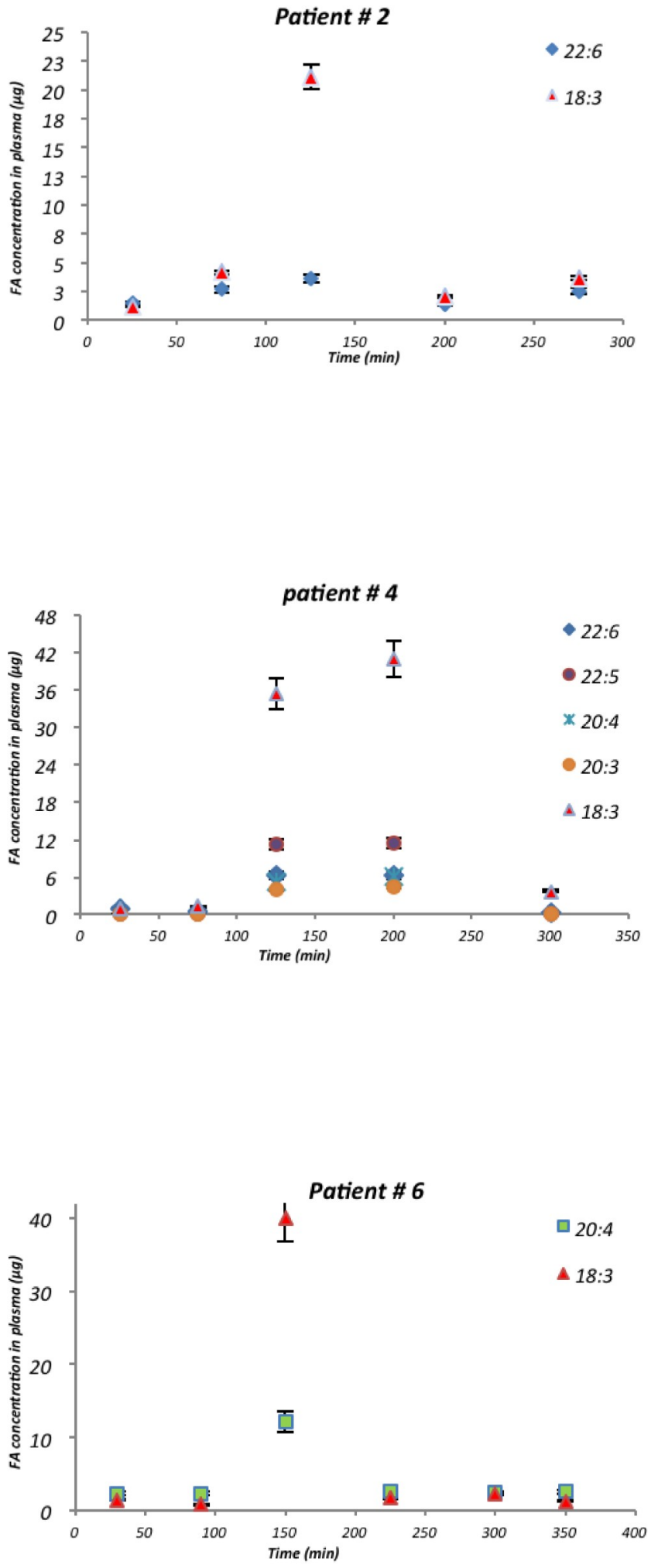


\section{CONCLUSION}

This research aims to develop a simple unbiased analytical method based on SPME in combination with LC-MS method for the extraction of free fatty acids from human and fish plasma. It was shown that the use of biocompatible C18 SPME fibers could be successfully applied for quantification and qualitative profiling of this group of lipids. This method permits the avoidance of interferences from hydrolysis of esterified fatty acids from other lipid sub-classes. Moreover, the protein affinity constant of polyunsaturated fatty acids has been determined and compared to the literature values. Indeed, the obtained results indicate the selectivity of the proposed method for the determination of fatty acid species despite their slight structural differences in carbon chain length and double bond number and localization. The extensive validation of the method demonstrates the fulfillment of requirements for the bioanalytical assays. The results of calibration equations obtained from fish and human plasma suggested that this approach could be extended to the plasma samples of other biological species. The application of the protocol allowed for the successful monitoring of the elevation of fatty acids in plasma collected from a group of patients undergone cardiac surgery right after initiation of extracorporeal circulation. The promising outcomes of the study show the potential of the approach for profiling and quantification of fatty acids in vivo in low invasive way as it was already demonstrated for other fiber-based SPME applications. ${ }^{28,31,68-71}$ The presented method could be also adopted for high throughput in vitro analysis either in its fiber form or using thin-film geometry format using automated 96-SPME device autosampler. ${ }^{72}$

\section{Supporting Information}

Additional information as noted in the text. This material is available free of charge via the Internet at http://pubs.acs.org.

\section{AUTHOR INFORMATION}

\section{Corresponding Author}

*Phone: +1-519-888-4641. Fax: +1-519-7460435. E-mail: janusz@ uwaterloo.ca.

\section{Present Addresses}

${ }^{\S}$ Emergency Science and Technology Section, Environment Canada; 335 River Road, Ottawa, Ontario, Canada $\mathrm{K} 1 \mathrm{~A} \mathrm{OH} 3$

\section{ACKNOWLEDGMENT}

The SPME study was supported by Natural Sciences and Engineering Research Council of Canada (NSERC). The authors express their sincere gratitude to Professor Mark Servos at Biology Department of University of Waterloo and the Environment Canada for providing fish plasma samples. Supelco is gratefully acknowledged for providing biocompatible C18 fibers. 


\section{REFERENCES}

(1) Van Bilsen, M.; van der Vusse, G. J.; Reneman, R. S. Pflugers Arch. 1998, 437, 2-14.

(2) Van der Vusse, G. J.; Glatz, J. F.; Stam, H. C.; Reneman, R. S. Physiol. Rev. 1992, 72, 881-940.

(3) Gao, X.; Li, K.; Hui, X.; Kong, X.; Sweeney, G.; Wang, Y.; Xu, A.; Teng, M.; Liu, P.; Wu, D. Biochem. J. 2011, 435, 723-732.

(4) Kahn, S. E.; Hull, R. L.; Utzschneider, K. M. Nature 2006, 444, 840-846.

(5) McGarry, J. D. Diabetes 2002, 51, 718.

(6) Novgorodtseva, T. P.; Karaman, Y. K.; Zhukova, N. V; Lobanova, E. G.; Antonyuk, M. V; Kantur, T. A. Lipids Health Dis. 2011, 10, 82.

(7) Zhou, Y.; Tian, C.; Jia, C. Br. J. Nutr. 2012, 108, 408-417.

(8) N.M. Weijers, R. Curr. Diabetes Rev. 2012, 8, 390-400.

(9) Lacaze, J.-P. C. L.; Stobo, L. A.; Turrell, E. A.; Quilliam, M. A. J. Chromatogr. A 2007, 1145, 51-57.

(10) Kopf, T.; Schmitz, G. J. Chromatogr. B. Analyt. Technol. Biomed. Life Sci. 2013, 938, 22-26.

(11) Hauff, S.; Vetter, W. Anal. Chim. Acta 2009, 636, 229-235.

(12) Pusvaskiene, E.; Januskevic, B.; Prichodko, A.; Vickackaite, V. Chromatographia 2008, 69, 271-276.

(13) Brondz, I. Anal. Chim. Acta 2002, 465, 1-37.
(14) Tsikas, D.; Zoerner, A.; Mitschke, A.; Homsi, Y.; Gutzki, F.-M.; Jordan, J. J. Chromatogr. B. Analyt. Technol. Biomed. Life Sci. 2009, 877, 28952908.

(15) Hellmuth, C.; Weber, M.; Koletzko, B.; Peissner, W. Anal. Chem. 2012, 84, 1483-1490.

(16) Kamphorst, J. J.; Fan, J.; Lu, W.; White, E.; Rabinowitz, J. D. Anal. Chem. 2011, 83, 9114-9122.

(17) Kallenbach, M.; Baldwin, I. T.; Bonaventure, G. Plant Methods 2009, 5, 17.

(18) J. Folch, M. Lees, G. H. S. S. J. Biol. Chem. 1957, 226, 497-509.

(19) Kotani, A.; Fuse, T.; Kusu, F. Anal. Biochem. 2000, 284, 65-69.

(20) Gutnikov, G. J. Chromatogr. B Biomed. Sci. Appl. 1995, 671, 71-89.

(21) Battistutta, F.; Buiatti, S.; Zenarola, C.; Zironi, R. J. High Resolut. Chromatogr. 1994, 17, 662-664.

(22) Péres, V. F.; Saffi, J.; Melecchi, M. I. S.; Abad, F. C.; de Assis Jacques, R.; Martinez, M. M.; Oliveira, E. C.; Caramão, E. B. J. Chromatogr. A 2006, 1105, 115-118.

(23) Bailey, A. L.; Southon, S. Anal. Chem. 1998, 70, 415-419.

(24) Simpson, N. J. K. Solid-Phase Extraction: Principles, Techniques, and Applications; CRC Press, 2000; p. 528.

(25) Poole, C. F. J. Chromatogr. A 885, 17 39.

(26) Ouyang, G. Anal. Chim. Acta 2008, 627, $184-197$. 
(27) Kataoka, H. J. Pharm. Biomed. Anal. 2011, 54, 926 - 950.

(28) Vuckovic, D.; Risticevic, S.; Pawliszyn, J. Angew. Chem. Int. Ed. Engl. 2011, 50, 5618-5628.

(29) Bojko, B.; Cudjoe, E.; Pawliszyn, J.; Wasowicz, M. TrAC Trends Anal. Chem. 2011, 30, 1505-1512.

(30) Bojko, B.; Reyes-Garcés, N.; Bessonneau, V.; Goryński, K.; Mousavi, F.; Souza Silva, E. a.; Pawliszyn, J. TrAC Trends Anal. Chem. 2014, 61, 168180.

(31) Bojko, B. Pawliszyn J. Bioanalysis 2014, 6, 1227-1239.

(32) Musteata, F. M.; Pawliszyn, J. TrAC Trends Anal. Chem. 2007, 26, 36-45.

(33) Ábalos, M.; Bayona, J. .; Pawliszyn, J. J. Chromatogr. A 2000, 873, 107115.

(34) Mitropoulou, A.; Hatzidimitriou, E.; Paraskevopoulou, A. Food Res. Int. 2011, 44, 1561-1570.

(35) Sharma, V.; Fan, J.; Jerath, a; Pang, K. S.; Bojko, B.; Pawliszyn, J.; Karski, J. M.; Yau, T.; McCluskey, S.; Wąsowicz, M. Anaesthesia 2012, 67, 12421250.

(36) Bojko, B.; Vuckovic, D.; Cudjoe, E.; Hoque, M. E.; Mirnaghi, F.; Wąsowicz, M.; Jerath, A.; Pawliszyn, J. J. Chromatogr. B 2011, 879, 3781-3787.

(37) Microextraction, S.; Bojko, B.; Vuckovic, D.; Mirnaghi, F.; Cudjoe, E.; Wasowicz, M.; Jerath, A.; Pawliszyn, J. 2012, 34, 31-37.

(38) Bojko, B.; Wąsowicz, M.; Pawliszyn, J. J. Pharm. Anal. 2014, 4, 6-13.
(39) Wąsowicz, M.; Jerath, A.; Bojko, B.; Sharma, V.; Pawliszyn, J.; McCluskey, S. Can. J. Anaesth. 2012, 59, 14-20.

(40) Ashbrook, J. D.; Spector, A. A.; Santos, E. C.; Fletcher, J. E. J. Biol. Chem. 1975, 250, 2333-2338.

(41) Wang, S.; Oakes, K. D.; Bragg, L. M.; Pawliszyn, J.; Dixon, G.; Servos, M. R. Chemosphere 2011, 85, 1472-1480.

(42) Musteata, F. M.; Pawliszyn, J. 2005, 789-800.

(43) Vuckovic, D.; Cudjoe, E.; Musteata, F. M.; Pawliszyn, J. Nat. Protoc. 2010, 5, 140-161.

(44) Mirnaghi, F. S.; Pawliszyn, J. J. Chromatogr. A 2012, 1261, 91-98.

(45) Vuckovic, D.; Pawliszyn, J. J. Pharm. Biomed. Anal. 2009, 50, 550-555.

(46) Mirnaghi, F. S.; Chen, Y.; Sidisky, L. M.; Pawliszyn, J. Anal. Chem. 2011, 83, 6018-6025.

(47) Bojko, B.; Vuckovic, D.; Pawliszyn, J. J. Pharm. Biomed. Anal. 2012, 66, 9199.

(48) Matuszewski, B. K.; Constanzer, M. L.; Chavez-Eng, C. M. Anal. Chem. 2003, 75, 3019-3030.

(49) Carrier, A.; Parent, J. J. Liq. Chromatogr. Relat. Technol. 2001, 24, 97-107.

(50) Risticevic, S.; Lord, H.; Górecki, T.; Arthur, C. L.; Pawliszyn, J. Nat. Protoc. 2010, 5, 122-139.

(51) Vuckovic, D.; Shirey, R.; Chen, Y.; Sidisky, L.; Aurand, C.; Stenerson, K.; Pawliszyn, J. Anal. Chim. Acta 2009, $638,175-185$. 
(52) Musteata, M. L.; Musteata, F. M.; Pawliszyn, J. Anal. Chem. 2007, 79, 6903-6911.

(53) Matuszewski, B. K.; Constanzer, M. L.; Chavez-Eng, C. M. Anal. Chem. 2003, 75, 3019-3030.

(54) Cavaliere, C.; Foglia, P.; Gubbiotti, R.; Sacchetti, P.; Samperi, R.; Laganà, A. Rapid Commun. Mass Spectrom. 2008, 22, 3089-3099.

(55) Van der VUSSE, G. J. Drug Metab. Pharmacokinet. 2009, 24, 300-307.

(56) Fredrickson, D. S.; Gordon, R. S. J. Clin. Invest. 1958, 37, 1504-1515.

(57) Hervé, F.; Urien, S.; Albengres, E.; Duché, J. C.; Tillement, J. P. Clin. Pharmacokinet. 1994, 26, 44-58.

(58) Stewart, A. .; Fujiwara, S.; Amisaki, T. Biochim. Biophys. Acta - Gen. Subj. 2013, 1830, 5427-5434.

(59) Musteata, F. M.; Pawliszyn, J.; Qian, M. G.; Wu, J.-T.; Miwa, G. T. J. Pharm. Sci. 2006, 95, 1712-1722.

(60) Brodersen, R.; Andersen, S.; Vorum, H.; Nielsen, S. U.; Pedersen, a O. Eur. J. Biochem. 1990, 189, 343-349.

(61) Department of Health and Human Services. Fed. Regist 2001, 66, 28526-28527.

(62) Mai, J.; Kinsella, J. E. J. Food Sci. 1979, 44, 1101-1105.

(63) Mai, J.; Kinsella, J. E. J. Food Biochem. 1980, 3, 229-239.

(64) Philibert, A.; Vanier, C.; Abdelouahab, N.; Chan, H. M.; Mergler, D. Am J Clin Nutr 2006, 84, 1299-1307.
(65) Tuunanen, H.; Ukkonen, H.; Knuuti, J. Curr. Cardiol. Rep. 2008, 10, 142148.

(66) Van der Vusse, G. J.; van Bilsen, M.; Glatz, J. F. Cardiovasc. Res. 2000, 45, 279-293.

(67) Turer, A. T.; Stevens, R. D.; Bain, J. R.; Muehlbauer, M. J.; van der Westhuizen, J.; Mathew, J. P.; Schwinn, D. A.; Glower, D. D.; Newgard, C. B.; Podgoreanu, M. V. Circulation 2009, $119,1736-1746$.

(68) Ouyang, G.; Vuckovic, D.; Pawliszyn, J. Chem. Rev. 2011, 111, 2784-2814.

(69) Musteata, F. M.; Pawliszyn, J. J. Biochem. Biophys. Methods 2007, 70, 181-193.

(70) Cudjoe, E.; Togunde, P.; Pawliszyn, J. 2012, 2605-2619.

(71) Bojko, B.; Gorynski, K.; Gomez-Rios, G. a; Knaak, J. M.; Machuca, T.; Cudjoe, E.; Spetzler, V. N.; Hsin, M.; Cypel, M.; Selzner, M.; Liu, M.; Keshjavee, S.; Pawliszyn, J. Lab. Invest. 2014, 94, 586-594.

(72) Vuckovic, D.; Cudjoe, E.; Hein, D.; Pawliszyn, J. 2010, 80, 6870-6880. 